On July Ioth, last, H. Kamerlingh Onnes finally succeeded in liquefying helium at the cryogenic laboratory of the University of Leiden (Akad. Welten. Amsterdam, pp. I63-79 (1908), and Comm. Phys. Lab. Leiden, No. I08). The method used was the counterpart of that already employed for hydrogen. The helium compressed to 50 to roo atm. was percooled to $15^{\circ}$ absolute by liquid hydrogen evaporating under a pressure of $6 \mathrm{~cm}$. of mercury and then allowed to expand in a small liquefier of the Hampson type. The liquid is so light (sp. gr. o. I5) that its liquefaction and separation from the gas was at first overlooked. Working with 2001 . of gas in the circulating system some $60 \mathrm{cc}$. of liquid were prepared. Its boiling point is about $4 \cdot 3^{\circ}$ absolute. Placed under a vacuum of $I$ $\mathrm{mm}$. of mercury no solidification or appreciable increase in viscosity took place, although the temperature of $3^{\circ}$ absolute was probably reached. How much lower we may go by increasing the degree of vacuum is yet an open question, but the author points out that should helium behave like pentane it would not become viscous above $1.5^{\circ}$ nor solid until $1.0^{\circ}$ absolute was reached. The critical temperature is estimated at not much above $5^{\circ}$ absolute and the critical pressure between 2 and 3 atmospheres.

BERRELEY, CAL.

\title{
THE EFFECT OF NEUTRAL SALTS ON HYDROLYSIS BY WATER.
}

By David R. Keliogg.

Received November $28,1908$.

[PRELIMINARY PAPER.]

It has long been known that when an ester undergoes hydrolysis in the presence of an acid, the addition of a neutral salt of the acid used, if the acid is a "strong" one, greatly increases the rate at which the reaction takes place. This was shown by Arrhenius, ${ }^{1}$ Euler, ${ }^{2}$ Spohr, ${ }^{3}$ and many others. In every case they found that the effect of the salt is greater the more dilute the acid. The question then arose as to the effect when the concentration of the acid is zero, $i . e$, in a neutral solution. The only work that has been discovered along this line was done by W. A. Smith." In his work on "The Progressive Dissociation of Organic Dibasic Acids," he determined the extent of dissociation by measuring the rate at which the acids inverted cane sugar. It occurred to him to investigate the effect of neutral salts on the rate of inversion in the absence of acid. Accordingly he tried solutions of sodium succinate, sodium oxalate, sodium sulphate, potassium chloride, and sodium carbonate, the solutions being I/64 normal. He found that salts of weak acids had almost no effect, while potassium chloride and sodium sulphate, which belong to the class of the most nearly neutral salts, had a large effect. The results for pure water and for potassium chloride he plotted on a time-per cent. inversion diagram-duplicate determinations varying for the potassium chloride,

\footnotetext{
1. $Z$. physik. Chem., 4, 237.

I Ibid., 32, 348.

3. j. prakt. Chem.. 33, 270.

1Z. physik. Chem., 25, 410
} 
by as much as 30.0 per cent. Smith merely mentions these results in passing, and considers the matter as of minor importance to the main subject of his investigation.

It was in the hope of throwing some light in this question, that, in the spring of 1907 , these experiments were carried on, the work being done at The Rhode Island College of Agriculture and Mechanic Arts. As a preliminary experiment, r.I225 g. of ethyl acetate were sealed in tubes containing $30.0 \mathrm{cc}$. respectively, of water, of a I.o per cent., a Io.o per cent., a 20.0 per cent. and of a saturated potassium chloride solution, the solutions being made to contain the given percentages by weight. The tubes had previously been thoroughly heated with dilute hydrochloric acid, with distilled water, and then repeatedly rinsed with distilled water. The sealed tubes containing the reaction mixture were then heated in an actively boiling water-bath for a definite time (about a day), cooled and the contents titrated with caustic soda and phenolphthalein. These results were, of course, not strictly comparable, as the amount of water in $30.0 \mathrm{cc}$. of the salt solutions was slightly less than for the same volume of pure water. However, they showed enough effect of the salt to warrant a more extended series of experiments. The results are given in Table $I$.

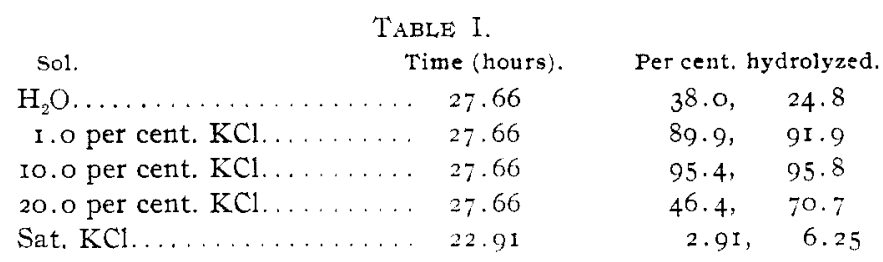

For the final experiments, volumes of the solutions were taken which would contain exactly $30.0 \mathrm{cc}$. of water, and, by means of a pipette made with a very narrow neck and stem, I.1225 g. of ethyl acetate added. A series was first run with pure water and ester, and then with the salt solutions, tubes being withdrawn at 2 -hour intervals, cooled and the contents titrated. The results are given in Table II.

It will be seen that the r.o per cent. solution exercised a very considerable accelerating effect, the ro.o per cent. solution a much larger one, and the 20.0 per cent. solution practically none. Table I shows that the saturated solution exercised a very strong inhibiting influence. The relation between concentration of the salt on the one hand and the acceleration produced thereby, on the other hand, is then, by no means a simple one, the effect passing through a maximum somewhere between r.o per cent. and 20.0 per cent. and changing sign somewhere between 20.0 per cent. and saturation. These results, while expressed quantitatively, may, perhaps, best be interpreted from a qualitative standpoint, as showing that neutral salts do exercise a very considerable effect on the 
TABLE II.

\begin{tabular}{|c|c|c|c|c|}
\hline \multirow[b]{2}{*}{ Time (hours). } & \multicolumn{4}{|c|}{ Per cent. hydrolyzed by } \\
\hline & water. & I per cent. $\mathrm{KCl}$. & ro per cent. $\mathrm{KCl}$. & 20 per cent. $\mathrm{KCl}$. \\
\hline 2.0 & 3.0 & 3.0 & 5.0 & I.O \\
\hline 4.0 & II.O & 1.3 .0 & . & $5 \cdot 0$ \\
\hline $4 \cdot 5$ & $\cdots$ & . & 34.0 & . \\
\hline 6.0 & I5.0 & $3 x .0$ & 42.0 & 9.0 \\
\hline 8.0 & 37.0 & 50.0 & 51.0 & 39.0 \\
\hline 10.0 & 48.0 & $55 \cdot 0$ & 73.0 & $49 \cdot 5$ \\
\hline I2.0 & 58.0 & 75.0 & 89.0 & 56.0 \\
\hline 14.0 & . & 80.0 & 89.0 & 70.0 \\
\hline 16.0 & . & 84.0 & 92.0 & 77.0 \\
\hline 18.0 & 78.0 & 90.0 & 92.5 & 80. \\
\hline 20.0 & 86.0 & . & 93.0 & 94.0 \\
\hline 22.0 & . & . & 96.0 & . \\
\hline 24.0 & 89.0 & 92.5 & . & 95.0 \\
\hline
\end{tabular}

rate of hydrolysis, even when no acid is present. They seem to warrant a more careful and extensive investigation of the subject, which is now in progress. I hope in the near future to be able to report on the relation between the rate of hydrolysis of ethyl acetate and ( $\mathrm{I}$ ) the concentration of the salt, (2) the nature of the salt, (3) the concentration of the ester itself, and (4) the temperature.

Physical laboratory, Ohto State University, November 23, 1908 .

\section{SOME OXIDATION PRODUCTS OF 6-NITRO-I,3-DIMETHYLBEN- ZENE-4-SULPHONIC ACID.}

By W. J. KARSLAKE AND P. A. BOND.

Received December 22, I908.

According to Harmsen, ${ }^{1}$ and Limpricht and Van Riesen, ${ }^{2}$ 6-nitro-I,3dimethylbenzene-4-sulphonic acid may be obtained either by the nitration of 1,3-dimethylbenzene-4-sulphonic acid or by the sulphonation of 4nitro-1,3-dimethylbenzene. Claus and Schmidt ${ }^{3}$ have also shown that while the nitration of $\mathrm{r}, 3$-dimethylbenzene-4-sulphonic acid yields the 6-nitro-r,3-dimethylbenzene-4-sulphonic acid as the principal product, yet the 2-nitro and the 5-nitro acids are formed at the same time, the relative quantities of each depending upon the conditions under which nitration takes place. In the preparation of the 6-nitro acid we essentially followed the directions of Claus and Schmidt except that ordinary commercial concentrated nitric acid was used instead of fuming nitric acid. The method used was as follows: Commercial metaxylene was shaken with an equal volume of commercial concentrated sulphuric acid

\footnotetext{
${ }^{1}$ Ber., 13, I 558.

Ibid., 18, 2191.

3 Ibid., 19, 1418.
} 